\title{
招請講演 I
}

\section{Advances in the Management of Patients with non-Hodgkin's Lymphoma}

\author{
James O. Armitage, M.D.
}

University of Nebraska Medical Center 983332 Nebraska Medical Center, Omaha, NE, USA

Non-Hodgkin's lymphoma represents a common malignancy and one that is increasing in frequency. Unfortunately, our treatment of patients with nonHodgkin's lymphomas over the last two decades has not lead to a major increase in the cure rate. However, knowledge regarding these malignancies is rapidly expanding, and provides the opportunity to improve treatment efficacy.

An important advance in the treatment of patients with non-Hodgkin's lymphoma is an improved understanding of the disorder itself. The REAL classification and the upcoming WHO classification, have incorporated our improved understanding of the non-Hodgkin's lymphomas. The new entities of mantle cell lymphoma, marginal zone lymphomas including MALT lymphoma and nodal marginal zone lymphoma, and anaplastic large T-/null cell lymphoma were previously included in other subgroups. These are distinct clinical/pathological entities with important therapeutic implications. Localized MALT lymphoma is curable with local therapies. Anaplastic large T-/null cell lymphoma has the best outlook of any large cell lymphoma. Mantle cell lymphoma has a poor outlook with any available therapy. Diffuse large B-cell lymphoma almost certainly includes several clinical entities. It maybe that some of these would be best treated with unique approaches. An important area of further research is identifying these subgroups.

A variety of new drugs and treatment approaches have recently come available for the management of patients with non-
Hodgkin's lymphoma. Although, used for several years, our knowledge of the use of high dose therapy and autologous or allogeneic bone marrow transplantation for treating patients with lymphoma continues to evolve. It is clear that allogeneic transplantation is associated with a significantly lower relapse rate, but a higher treatment related death rate. Autologous transplantation is the treatment of choice for patients with relapsed diffuse aggressive lymphoma. Its use earlier in the treatment of patients with high risk diffuse aggressive lymphoma or in patients with follicular lymphoma or in patients with tollicular lymphoma continues to be studied. A number of new agents are also now available including drugs such as fludarabine and biologically active molecules such as interferon and rituximab. Fludarabine is the most active drug in small lymphocytic lymphoma/chronic lymphocytic leukemia. Interferon prolongs remission duration and, possibly, survival in patients with follicular lymphomas. Its place in aggressive lymphomas is now being considered. Rituximab causes responses in both follicular lymphoma and diffuse large Bcell lymphoma. The development of a strategy for its optimal use is important.

The treatments available for the management of patients with non-Hodgkin's lymphoma continue to expand. We need to develop the knowledge for their optimal use. Hopefully, this will lead to a higher overall survival for patients suffering from these malignancies. 
招請講演 II

\title{
ALK-POSITIVE LYMPHOMA
}

\author{
David Y. Mason \\ Professor of Cellular Pathology, Nuffield Department of Clinical Biochemistry and \\ Cellular Science, John Radcliffe Hospital, Oxford, OX3 9DU, UK.
}

For many years pathologists have sought immunocytochemically detectable proteins which can be used as specific markers of malignant cells. One approach to the identification of such markers is to raise antibodies to the protein products of genes that are rearranged, and which are therefore likely to be directly implicated in oncogenesis. A widely studied marker of this sort is the product of the $B C L-2$ gene, rearranged by the $(14 ; 18)$ translocation in follicular lymphoma. This protein is specific for neoplastic germinal centre cells, since they lack this marker unless they have transformed to create a follicular lymphoma. However, BCL-2 protein is widely expressed in non-neoplastic lymphoid cells outside the germinal centre, limiting its value as a practical diagnostic marker.

A much more specific marker is the ALK protein. Many cases of anaplastic large cell lymphoma show the $(2 ; 5)$ chromosomal translocation. Work in Japan and the United States showed that this creates a fusion gene encoding the hybrid NPMALK protein, in which the N-terminal position of nucleophosmin is fused to the intracytoplasmic portion of the ALK tyrosine kinase. This protein undergoes spontaneous dimerisation leading to phosphorylation of intracytoplasmic proteins and malignant transformation.

Identification of ALK protein by immunohistochemistry is now widely used as a simple means of identifying lymphomas carrying the $t(2 ; 5)$ translocation. It is also useful for the recognition of minimal infiltrates, for example in bone marrow and lymph nodes. Immunostaining for ALK has led to a re-evaluation of the diagnostic criteria for anaplastic large cell lymphoma. Many haematopathologists today identify "ALK-positive" lymphoma (or "ALK-oma") as a specific entity which tends to have the following features :

- occurrence in the first two decades of life

- cytotoxic $T$ cell phenotype

- wide morphologic spectrum ranging from small to large cell

- good prognosis.

The study of ALK-positive lymphoma has opened a number of new avenues. It is now clear that $10-20 \%$ of these neoplasms carry variants of the $(2 ; 5)$ translocation in which ALK is linked to a protein other than NPM. These alternative partners for ALK may prove to be involved in the genesis of tumours other than lymphoma. Furthermore, the fact that ALK protein is highly restricted in its expression in normal tissue (being found only in a minority of cells in the central nervous system) means that it may elicit an immune response which could, if augmented by specific antigenic stimulation, be of therapeutic benefit. 